\title{
Bax dimerizes via a symmetric BH3:groove interface during apoptosis
}

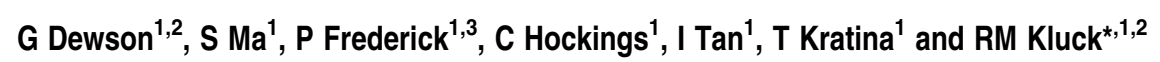

During apoptotic cell death, Bax and Bak change conformation and homo-oligomerize to permeabilize mitochondria. We recently reported that Bak homodimerizes via an interaction between the BH3 domain and hydrophobic surface groove, that this BH3:groove interaction is symmetric, and that symmetric dimers can be linked via the $\alpha 6$-helices to form the high order oligomers thought responsible for pore formation. We now show that Bax also dimerizes via a BH3:groove interaction after apoptotic signaling in cells and in mitochondrial fractions. BH3:groove dimers of Bax were symmetric as dimers but not higher order oligomers could be linked by cysteine residues placed in both the $\mathrm{BH} 3$ and groove. The BH3:groove interaction was evident in the majority of mitochondrial Bax after apoptotic signaling, and correlated strongly with cytochrome $c$ release, supporting its central role in Bax function. A second interface between the Bax $\alpha 6$-helices was implicated by cysteine linkage studies, and could link dimers to higher order oligomers. We also found that a population of Bax:Bak heterodimers generated during apoptosis formed via a BH3:groove interaction, further demonstrating that Bax and Bak oligomerize via similar mechanisms. These findings highlight the importance of BH3:groove interactions in apoptosis regulation by the Bcl-2 protein family.

Cell Death and Differentiation (2012) 19, 661-670; doi:10.1038/cdd.2011.138; published online 21 October 2011

Bax and Bak are pivotal effectors of the intrinsic or mitochondrial apoptosis pathway, as either Bax or Bak is needed to permeabilize the mitochondrial outer membrane (MOM). ${ }^{1,2}$ Accordingly, the two proteins exhibit significant sequence (Figure 1a) and structural homology. ${ }^{3,4}$ Aspects of Bax and Bak regulation are distinct, however, as Bak is integrated into the MOM in healthy cells, whereas Bax is predominantly cytosolic and translocates to mitochondria after an apoptotic stimulus. ${ }^{5,6}$ Furthermore, Bax and Bak are countered by different pro-survival $\mathrm{Bcl}-2$ proteins. ${ }^{7,8}$

To form the apoptotic pore in the MOM, both Bax and Bak undergo conformation change, including exposure of $\mathrm{N}$-terminal epitopes, that allows them to homo-oligomerize., ${ }^{6,9-11}$ Bax conformation change also involves insertion of $\alpha 5$ and $\alpha 6$ helices into the MOM. ${ }^{12}$ We recently reported that Bak activation requires exposure of the $\mathrm{BH} 3$ domain, which then binds to the hydrophobic surface groove of another activated Bak molecule to form homodimers. ${ }^{13}$ Reciprocity of the $\mathrm{BH}$ 3:groove interaction generates symmetric dimers that can be linked via a second interface between $\alpha 6$-helices to form the higher order oligomers thought necessary to permeabilize the MOM. ${ }^{14}$

In Bax, a role for the $\mathrm{BH} 3$ domain in oligomerization and pro-apoptotic function was implicated by mutagenesis studies. ${ }^{15,16}$ More recently, in liposomes Bax formed symmetric dimers via the $\mathrm{BH} 3$ domains, as detected by electron paramagnetic resonance spectroscopy. ${ }^{17}$ In addition, in Bax oligomers induced by the detergent Triton $\mathrm{X}-100, \mathrm{BH} 3$ domain residues could cross-link to another Bax molecule, although the receptor surface was not identified. ${ }^{18}$ Thus, it remains unclear how Bax oligomerizes within the MOM during apoptosis, including whether the $\mathrm{BH} 3$ domain binds to the hydrophobic groove.

Here we use cysteine linkage and blue native PAGE (BN-PAGE) to examine how Bax oligomerizes at the MOM following apoptotic signaling. We show that symmetric $\mathrm{BH} 3$ :groove dimers of Bax form within the MOM following etoposide treatment of cells, and following truncated Bid (tBid) -treatment of mitochondria. The symmetric dimers can be linked via a second interface at $\alpha 6$ to form higher order complexes. Heterodimers of Bax and Bak also form via a $\mathrm{BH}$ :groove interaction.

\section{Results}

Bax conformation change during apoptosis reflects that of Bak. To monitor Bax conformation change, we first considered whether linkage of endogenous cysteines could identify different Bax conformations, as we and others have reported for Bak. ${ }^{13,14,19}$ For example, in non-activated Bak, C14 and C166 form an intramolecular disulfide bond when oxidant is added $\left(\mathrm{M}_{\mathrm{x}}\right.$; Figure $\left.1 \mathrm{~b}\right)$, whereas after etoposide C14 and C166 reposition to allow linkage of oligomers

\footnotetext{
${ }^{1}$ The Walter and Eliza Hall Institute of Medical Research, Parkville, Victoria, Australia and ${ }^{2}$ Department of Medical Biology, The University of Melbourne, Parkville, Victoria, Australia

*Corresponding author: RM Kluck, The Walter and Eliza Hall Institute of Medical Research, 1G Royal Parade, Parkville 3050, Australia. Tel: +61 39345 2487;

E-mail: kluck@wehi.edu.au

${ }^{3}$ Current address: Department of Biochemistry, Monash University, Melbourne, 3800, Australia

Keywords: apoptosis; Bax; $\mathrm{BH} 3$ domain; heterodimer; oligomer; mitochondria

Abbreviations: BH3, Bcl-2 homology 3; BN-PAGE, blue native PAGE; CuPhe, copper(II)(1,10-phenanthroline) $)_{3}$; DTT, dithiothreitol; GFP, green fluorescent protein; HA, haemagglutinin; hBak, human Bak; hBax, human Bax; IRES, internal ribosome entry site; LOF, loss-of-function; MEFs, mouse embryonic fibroblasts; MOM, mitochondrial outer membrane; tBid, truncated Bid; TNF- $\alpha$, tumour necrosis factor alpha; wt, wild-type

Received 11.3.11; revised 08.8.11; accepted 20.8.11; Edited by R Youle; published online 21.10.11
} 
a

Bax mdgSgeqprgggptsseqimktqalliqqfigdragrmggea-------pel 45

Bak masgqgpgpprqeCgepalpsaseeqvaqdteevfrsyvfyrhqgeqeaegvaapadpem 60

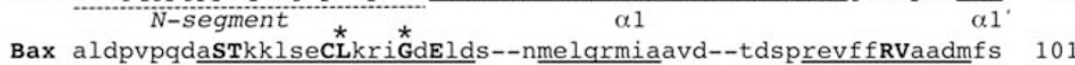

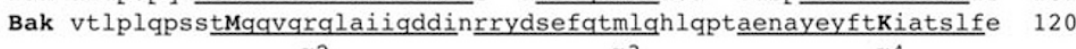

$\alpha 2 \quad \alpha 3 \quad \alpha 4$

Bax dgnfnwqrvvalfyfasklvlkalctkvpelirtimqwtldflreRLlq-wiqdqggwdql 161

Bak sg-inwgrvvallqfqyrlalhvyqhgltgflqqvtrfvvdfmlhhciarwiagrggwvaa 180

$\alpha 5$
$\alpha 6$

Bax lsyfgtptwatvtifvagvltasltiwkkmg 192

Bak $\frac{\text { lnlgngpilnvlvvl-gvvllgqfvvreffks } 211}{\alpha 9}$

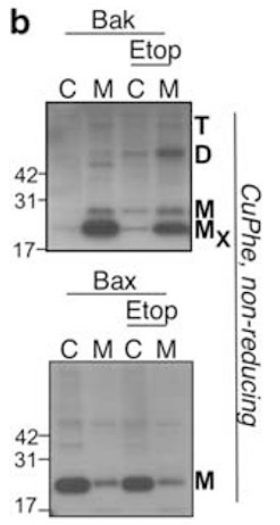

C

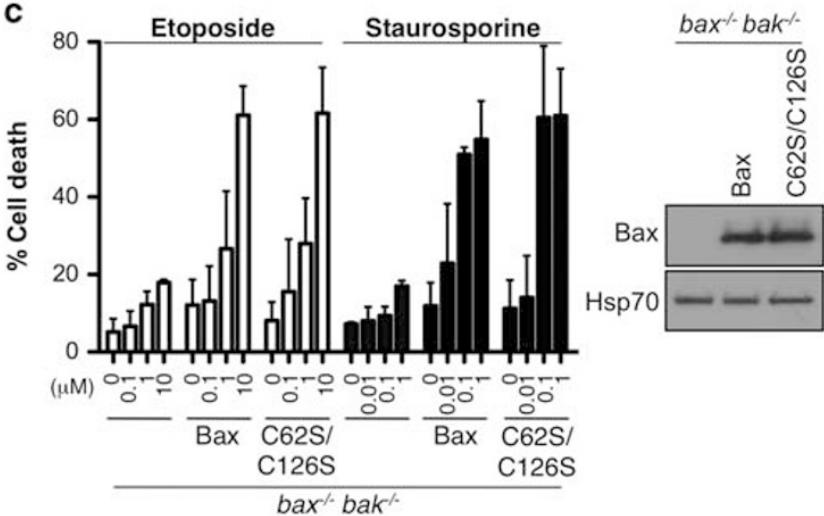

d

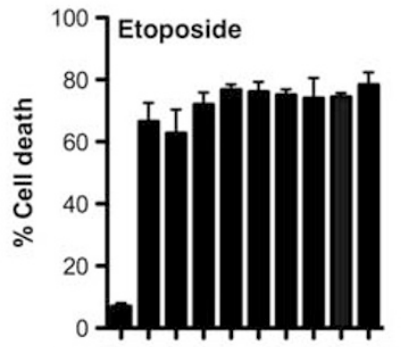

$\begin{array}{ll}\text { e } 10 \mathrm{C} / & \mathrm{G} 10 \mathrm{C} / \\ \mathrm{L} 148 \mathrm{C} & \mathrm{R} 147 \mathrm{C}\end{array}$

$\alpha 7 \quad \alpha 8$

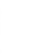


re-introduced in Cys-null Bax at S4 or G10 and L148 or R147. Each variant was expressed at levels similar to that of wildtype Bax, and retained pro-apoptotic function (Figure 1d). Before apoptosis, the two introduced cysteines were nearby in the same Bax molecule as oxidant induced intramolecular linkage $\left(\mathrm{M}_{\mathrm{x}}\right.$; Figure $1 \mathrm{e}$ and Supplementary Figure 1). Efficiency of intramolecular linkage was lower in Bax than in Bak $\left(\mathrm{M}_{\mathrm{x}}\right.$; Figure $\left.1 \mathrm{~b}\right)$, perhaps owing to sub-optimal cysteine positions, in particular within the Bax $\mathrm{N}$-segment, as this region in Bax and Bak differs significantly in sequence (Figure 1a), and its absence in the Bak structure precludes structural alignment. ${ }^{3}$ Inefficient intramolecular linkage in Bax may also be explained by flexibility of the Bax $\mathrm{N}$-segment ${ }^{4}$ compared with the Bak $\mathrm{N}$-segment. ${ }^{14}$ Nevertheless, the observed intramolecular linkage indicates that the $\mathrm{N}$-segment and $\alpha 6$ regions are close in the non-activated forms of Bax and Bak.

After apoptosis, the Bax $\mathrm{N}$-segment and $\alpha 6$ regions had repositioned, as after oxidant dimers and trimers were evident in the double-cysteine variants (Figure 1e and Supplementary Figure 1). Dimers and trimers were only detected in mitochondrial fractions suggesting that Bax converts from a monomer in cytosol to an oligomer once at mitochondria, as reported previously. ${ }^{22,23}$ Single-cysteine variants could also link to dimers after apoptosis (Figure 1e), indicating that the $\mathrm{N}$-segments and $\alpha 6$ regions can each self-associate in oligomerized Bax, as observed for oligomerized Bak. ${ }^{14}$

In summary, by introducing two cysteines in Bax at the cysteine positions in Bak and monitoring their ability to form disulfide bonds, we show that Bax and Bak adopt similar conformations before apoptosis, and again after apoptosis.

Bax forms BH3:groove homodimers during apoptosis. We next tested whether Bax oligomerization involves a $\mathrm{BH} 3:$ groove interaction. Cysteine was introduced in Cys-null Bax at positions predicted to be in close proximity in a BH3:groove interaction (Figure 2a). Cysteine substitutions were also generated in a mitochondria-targeted form of Bax, S184L. ${ }^{24}$ Each single-cysteine $\mathrm{BH} 3$ and groove variant (bearing FLAG or HA epitope tags at the $\mathrm{N}$ terminus) retained pro-apoptotic function (Figure $2 b$ ). To test for a $\mathrm{BH}$ :groove interaction, single-cysteine variants were co-expressed, apoptosis was initiated, and disulfide bonding was induced. FLAG variants were then immunoprecipitated from membrane fractions and blotted for the associated HA-variant (Figure 2c, top panel). When run under nonreducing conditions the co-precipitated $\mathrm{HA}$-variant was evident either as a monomer, or as a dimer linked to the FLAG-variant. Notably, linkable dimers were generated if $\mathrm{BH} 3$ and groove cysteine variants were co-expressed, but not if two $\mathrm{BH} 3$ variants were co-expressed (Figure 2c), indicating that a $\mathrm{BH} 3$ :groove interaction is present in oligomerized Bax. Dimers were again absent in cytosolic fractions (data not shown).

A Bax BH3:groove interaction was also tested using mitochondria isolated from cells expressing the mitochondria-targeted BaxS184L. ${ }^{24}$ Incubation with tBid triggered Bax activation and MOM permeabilization (see below), and provided a model corresponding to that used for Bak. ${ }^{13}$ tBid treatment induced linkable dimers if $\mathrm{BH} 3$ and groove cysteine variants were co-expressed, but not if two $\mathrm{BH} 3$ variants were co-expressed (Figure 2c). A nearby BH3 cysteine, S55C, showed a similar linkage pattern (Supplementary Figure 2), while cysteine introduced at a more distal position in the $\mathrm{BH} 3$, $\mathrm{E} 69 \mathrm{C}$, was unable to link to either groove cysteine (Supplementary Figure 3), as predicted (Figure 2a). Notably, near $100 \%$ linkage occurred when the T56C or S55C and R94C variants were co-expressed, indicating that all mitochondrial Bax had adopted a BH3:groove interaction. As the equivalent pairing in Bak (M71C:K113C; Figure 1a) also yielded near $100 \%$ dimers in response to $\mathrm{HBid}^{13}{ }^{13}$ the $\mathrm{BH} 3$ :groove interactions in both proteins appear equivalent at the molecular level. The BH3:groove interaction occurred only after Bax had become activated, as disulfide-linkable dimers were absent before tBid treatment (Figure 2e).

The BH3:groove interaction in Bax dimers is symmetric. To test whether the Bax BH3:groove homodimer is symmetric, as we have shown for the Bak homodimer, ${ }^{14}$ we generated $\mathrm{BH} 3$ :groove double-cysteine variants (S55C/R94C and T56C/R94C) of Bax and of BaxS184L. A symmetric BH3:groove interaction (Figure 3a) predicts that cysteine linkage would generate dimers but not trimers or higher order oligomers.

The double-cysteine variants in Bax and in BaxS184L retained normal function (Figure $3 b$ ). They also oligomerized like wild-type Bax when analyzed by BN-PAGE (Figure 3c), a technique that utilizes digitonin to solubilize membrane proteins without denaturing proteins or disrupting supramolecular complexes. ${ }^{25,26}$ For example, before apoptosis, Bax variants in the cytosolic fraction migrated at low molecular weight, consistent with cytosolic Bax being monomeric, ${ }^{5}$ while Bax variants in the membrane fraction were either monomeric or within a high molecular weight complex, as observed in HeLa cells. ${ }^{26}$ After apoptosis, each Bax variant formed a ladder in the membrane fraction, similar to those observed in TNF- $\alpha$-treated HeLa cells. ${ }^{26}$ Thus, the double-cysteine substitutions did not alter Bax pro-apoptotic function or oligomerization.

When assessed by cysteine linkage, the double-cysteine Bax variants formed linkable dimers after etoposide treatment (Figure 3d). As for the single-cysteine variants in Figure 2, dimers were only in the mitochondrial fractions, with essentially all mitochondrial Bax forming a BH3:groove dimer. Notably, trimers and higher order oligomers were not observed despite the presence of two cysteines in each protein. For comparison, the two cysteines in Bax $\mathrm{G} 10 \mathrm{C} /$ $\mathrm{R} 147 \mathrm{C}$ could link to complexes larger than dimers (Figure 3d). As $\mathrm{BH} 3$ :groove linkage of single-cysteine variants was near complete (Figure $2 \mathrm{~d}$ ), yet linkage of double-cysteine variants generated only dimers (Figure $3 d$ ), these data argue that the $\mathrm{Bax} \mathrm{BH} 3$ :groove interaction is reciprocal, as predicted by symmetric dimers (Figure 3a).

When tested following tBid treatment of mitochondrial fractions, the double-cysteine BaxS184L variants were also able to link only as dimers (Figure $3 e$ ). In addition, dimer formation correlated with cytochrome $c$ release, strongly supporting a role for Bax BH3:groove dimers in pore formation.

To further assess the physiological relevance of the $\mathrm{BH} 3:$ groove interaction we tested whether loss-of-function 
a

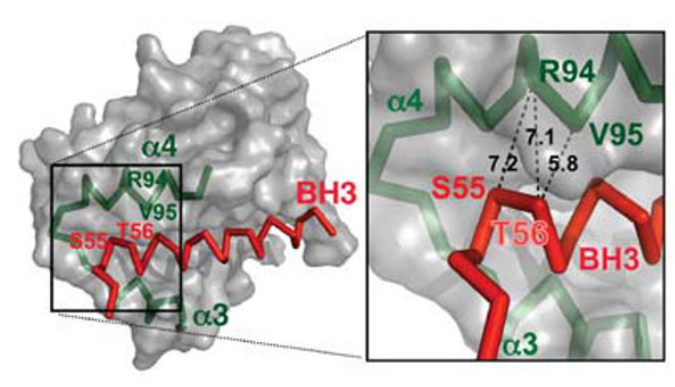

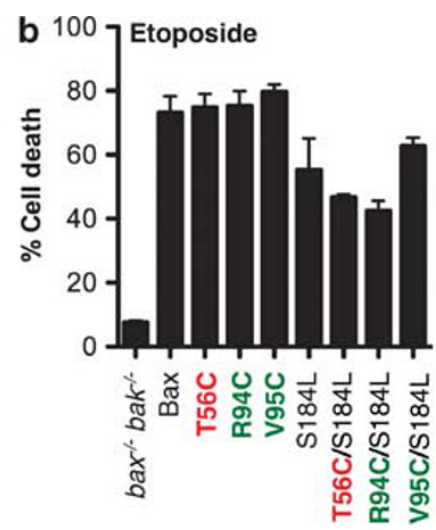

C

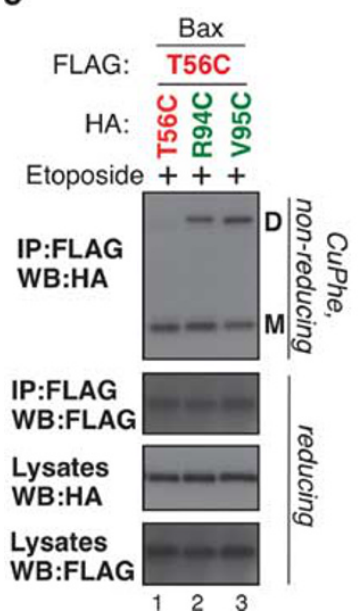

d

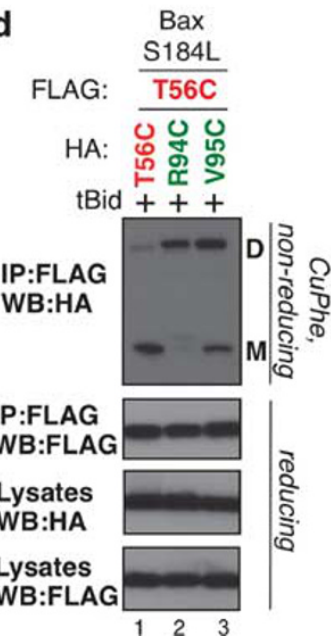

e

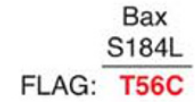

$\mathrm{Bax}$
$\mathrm{S} 184 \mathrm{~L}$

FAG: T56C

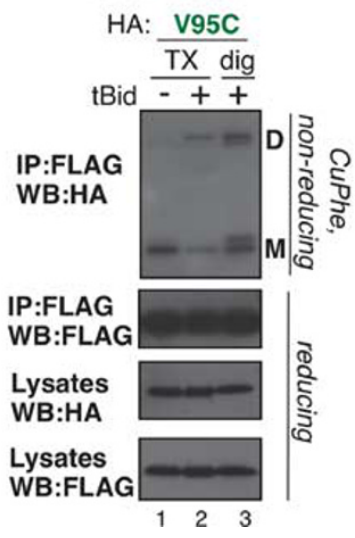

Figure 2 Bax homodimerizes via a BH3:groove interaction during apoptosis. (a) Model of BH3:groove interaction in Bax dimers. A model of a BH3 domain (based on Bim; red) bound to the Bax hydrophobic groove ( $\alpha 3$ and $\alpha 4$; aa74-100; green) is based on an alignment of the Bax and Bcl- $\mathrm{x}_{\mathrm{L}}:$ Bim structures. ${ }^{4,40}$ Residues mutated to cysteine and the predicted molecular distances (A) between them are indicated. (b) Bax BH3 and groove cysteine variants retain apoptotic function. bax ${ }^{-1-}$ bak $^{-/-}$MEFs expressing the indicated Bax variants were treated with etoposide $(10 \mu \mathrm{M})$. Cell death is expressed as mean \pm S.D. of three independent experiments. (c) Bax BH3 residues are juxtaposed with $\alpha 4$ residues after etoposide treatment. Cells expressing pairs of FLAG- and HA-tagged single-cysteine Bax variants were treated with etoposide (10 $\mu \mathrm{M})$. Mitochondrial fractions were incubated with CuPhe, solubilized in 1\% Triton X-100 and immunoprecipitated for FLAG before non-reducing or reducing SDS-PAGE. Immunoprecipitates (IP) and cell lysates were western blotted (WB) for HA or FLAG, as indicated. Data are representative of two independent experiments. (d) Bax BH3 residues are juxtaposed with $\alpha 4$ residues after tBid treatment. Membrane fractions from MEFs expressing BaxS184L BH3 and groove cysteine variants were treated with tBid and examined as in (c). Data are representative of two independent experiments. (e) BH3:groove interaction is specific for activated Bax. Membrane fractions from MEFs expressing BaxS184L BH3 and groove cysteine variants were treated with or without tBid before induction of disulfide-linkage, solubilization in either $1 \%$ Triton X-100 (TX) or $1 \%$ digitonin and immunoprecipitation as in (c). Data are representative of two independent experiments

(LOF) mutations in the Bax $\mathrm{BH} 3$ domain precluded disulfidelinkage of the BH3:groove interface. Bax L63A and G67K failed to function when expressed in $b^{-1-} \mathrm{bak}^{-/-} \mathrm{MEFs}$ (Figure 4a), and failed to oligomerize after etoposide as assessed by BN-PAGE and by BH3:groove linkage (Figure 4b). However, as these variants also failed to translocate to mitochondria after etoposide (Figure 4b), the mutations appear to block Bax activation, consistent with a previous study. ${ }^{23}$ To bypass the step needed for etoposideinduced Bax activation we used Triton X-100 to induce Bax oligomerization. Notably, cell lysis in Triton X-100 (but not in digitonin) allowed BH3:groove linkage of double-cysteine Bax, but not if the $\mathrm{BH} 3$ was mutated (Figure 4c). In another test of physiological relevance, $\mathrm{Bcl}-2$ expression blocked both Bax BH3:groove linkage and cytochrome $c$ release (Figure 4d). These findings each support the importance of the BH3:groove interaction in Bax apoptotic function.
Bax BH3:groove dimers are symmetric and can be linked via an $\alpha 6: \alpha 6$ interface. Oligomerized Bax exhibited linkage between the $\mathrm{N}$ termini (e.g., G10C:G10C) and between the a6-helices (e.g., R147C:R147C) in Figure 1e suggesting that these interfaces may be important for Bax oligomerization and function. However, truncating 5 or 10 residues did not affect apoptotic function or oligomerization, indicating that an $\mathrm{N}$-terminal interface is not important for Bax function (Supplementary Figure 4). The observed $\mathrm{N}$-terminal linkage may be due to the flexibility of the $\mathrm{N}$-segment as indicated by the structure of non-activated $\mathrm{Bax}^{4}$ and by exposure of the $\mathrm{N}$-terminal 6A7 epitope in activated Bax and Bax G10C (Supplementary Figure $4 c)^{6}$

To test whether an interface between the $\alpha 6$ helices is involved in Bax oligomerization, we introduced single cysteines in $\alpha 6$, either along the surface or at 1136 that orientates toward the core in non-activated Bax (Figure 5a), ${ }^{4}$ 
a
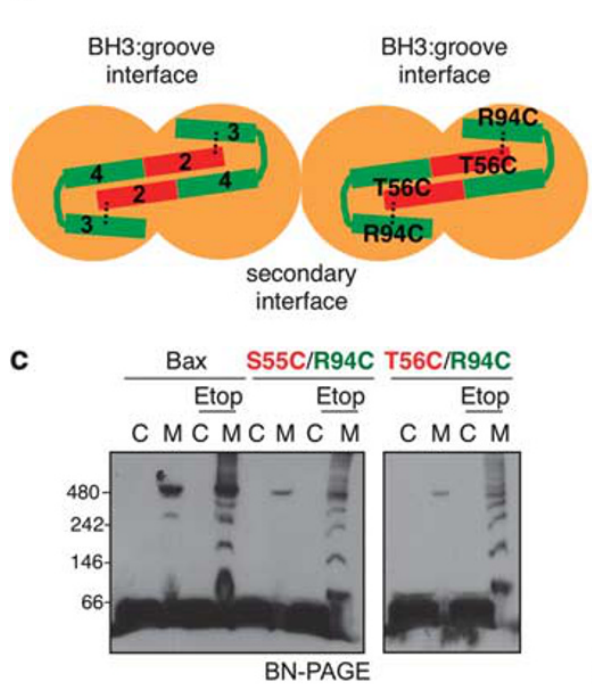

d

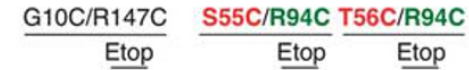

b ${ }^{80}$ ]Etoposide

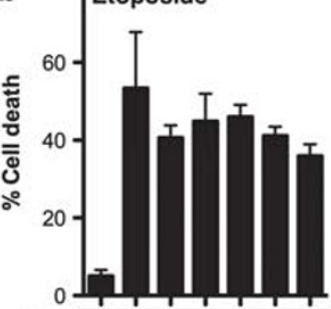

Bax

HSP70

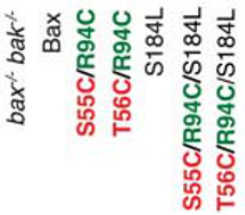

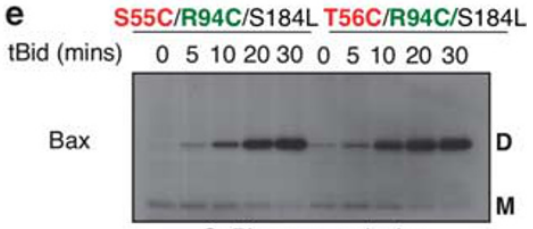

CuPhe, non-reducing

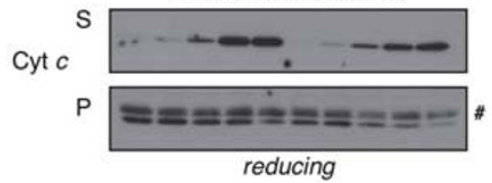

Figure 3 Bax BH3:groove dimers are symmetric and their formation correlates with cytochrome $c$ release. (a) Schematic of Bax oligomerization. Symmetric dimer formed by reciprocal BH3:groove interactions with $\alpha$-helices 2-4 indicated (left). Bax with cysteine in the $\mathrm{BH} 3$ and in the groove (T56C/R94C) would form disulfide bonds within a dimer, but not between dimers (right). Symmetric dimers may link via a secondary interface (e.g. between the $\alpha 6$-helices; Figure 1e) to form higher order oligomers. (b) BH3/groove double-cysteine variants retain apoptotic function. bax ${ }^{-1-}$ bak $^{-/-} \mathrm{MEFs}$ expressing the indicated Bax variants were treated with etoposide $(10 \mu \mathrm{M})$. Cell death is expressed as mean \pm S.D. of three independent experiments. (c) BH3/groove double-cysteine variants oligomerize like wild-type Bax on BN-PAGE. MEFs expressing Bax or the indicated cysteine variants were treated with or without etoposide, and cytosol $(C)$ and membrane $(M)$ fractions generated. Fractions were solubilized in $1 \%$ digitonin, analyzed by BNPAGE and immunoblotted for Bax. Data are representative of three independent experiments. (d) BH3/groove double-cysteine variants form disulfide-linkable dimers but not higher order oligomers. Cells expressing the indicated Bax variants were left untreated or treated with etoposide $(10 \mu \mathrm{M})$. Cytosol $(\mathrm{C})$ and membrane $(\mathrm{M})$ fractions were treated with CuPhe and analyzed as in Figure $1 \mathrm{~b}$. Note that a band slightly smaller than dimer size ( $\left.{ }^{*}\right)$ in the cytosolic T56C/R94C fractions does not relate to apoptosis as it occurs before and after etoposide treatment. Data are representative of two independent experiments. (e) Formation of Bax BH3:groove dimers correlates with cytochrome $c$ release. Membrane fractions from cells expressing BaxS184L variants were treated with tBid for the indicated times prior to CuPhe addition and analysis as in (d) (top panel). Alternatively, samples were separated into supernatant $(\mathrm{S})$ and membrane $(\mathrm{P})$ fractions and assessed for cytochrome $c$ (Cyt $c$; bottom panels). "Non-specific band. Data are representative of two independent experiments

and tested whether each cysteine could disulfide-link to the same residue in another Bax molecule after apoptosis. Each $\alpha 6$ cysteine mutant retained apoptotic function (Figure $5 b$ ). After etoposide, disulfide-linkable dimers were particularly evident in W139C and E146C in the membrane fractions (Figure 5c,

top panels). Linkage of these $\alpha 6$ residues, and of $\mathrm{R} 147 \mathrm{C}$ in Figure 1e, indicates that the $\alpha 6$-helices are in close proximity in a Bax oligomer.

We then tested whether the $\alpha 6: \alpha 6$ interface could link symmetric dimers to form high order multimers of Bax, as we had found for Bak. ${ }^{14}$ Triple-cysteine mutants were generated to allow linkage at both the BH3:groove (S55C:R94C) and $\alpha 6: \alpha 6$ (e.g., E146C:E146C) interfaces, with each variant retaining apoptotic function (Figure $5 \mathrm{~b}$ ). Consistent with our hypothesis, disulfide linkage trapped higher order complexes of $\mathrm{Bax}$ in the membrane of etoposide-treated cells, again particularly with the W139C and E146C variants (Figure 5c, bottom panels). The disulfide-linked oligomers converted to monomers when run under reducing conditions (Supplementary Figure 5a), confirming that the complexes were disulfidelinked Bax. Two features of the linkage pattern indicate that $\mathrm{BH} 3:$ groove dimers are linked by an $\alpha 6: \alpha 6$ interface. First, the dimer in the triple-cysteines is a BH3:groove dimer, running slightly slower than an $\alpha 6: \alpha 6$ dimer (Supplementary Figure $5 b)$. Second, the absence of trimers indicates that the BH3:groove dimers are symmetric, with cysteines in an alternative interface necessary to link multimers.

Heterodimers of Bax and Bak also contain a BH3:groove interaction, but form a minor population in apoptotic cells. Given the conserved BH3:groove mechanism of homodimer formation in Bax and Bak, we tested whether Bak and Bax also heterodimerize via a BH3:groove 


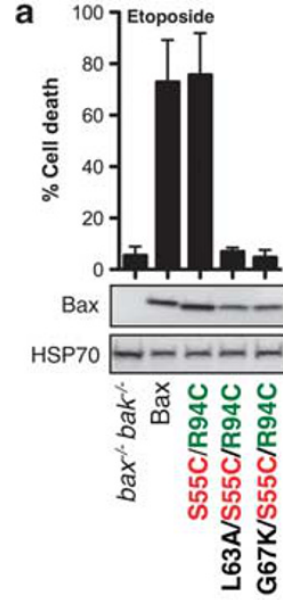

b

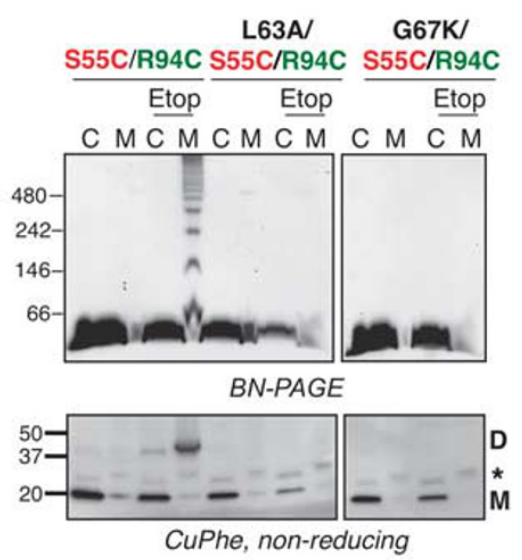

C

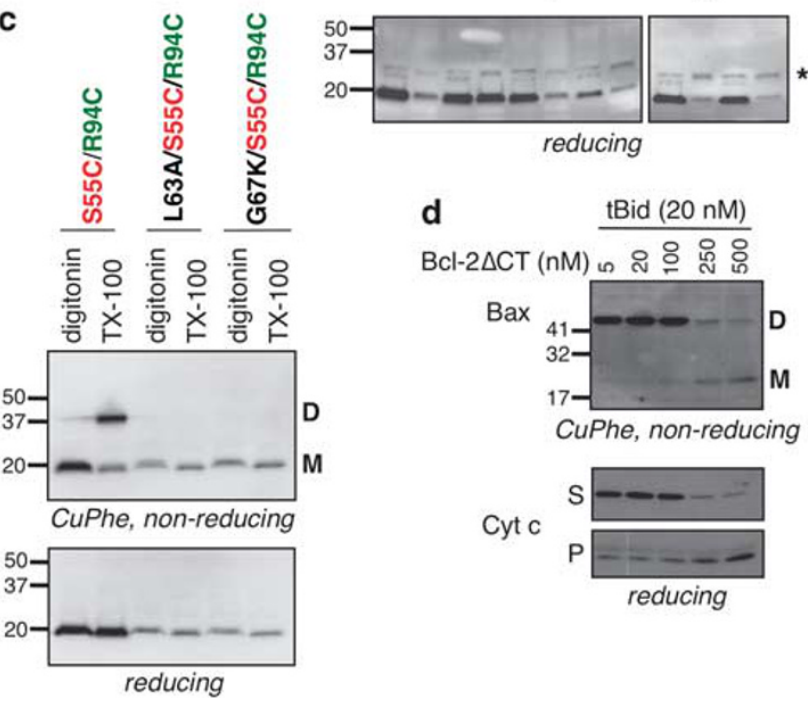

Figure 4 Bax $\mathrm{BH}$ :groove interaction correlates with apoptotic function. (a) Mutation of key residues in the Bax BH3 domain disrupts function. bax ${ }^{-/}$bak ${ }^{-/-}$ MEFs stably expressing the indicated Bax variants were assessed for protein expression (lower panels), and for cell death following treatment with etoposide $(10 \mu \mathrm{M})$. Cell death is expressed as mean \pm S.D. of three independent experiments. (b) Loss-of-function Bax $\mathrm{BH} 3$ mutants fail to oligomerize. MEFs expressing the indicated Bax variants were left untreated or treated with etoposide $(10 \mu \mathrm{M})$. Cytosol (C) and membrane (M) fractions were separated and either solubilized in $1 \%$ digitonin for BN-PAGE, or treated with CuPhe and analyzed by SDS-PAGE under non-reducing or reducing conditions. Data are representative of two independent experiments. Note that the BH3:groove disulfide-linked dimer is abolished under reducing conditions, while the indicated bands $\left({ }^{*}\right)$ were not disulfide-linked Bax complexes as they were resistant to reduction. (c) $\mathrm{BH} 3$ :groove interaction induced by detergent is blocked by mutation of the $\mathrm{BH} 3$ domain. Cells expressing the indicated Bax variants were lysed in $1 \%$ Triton $\mathrm{X}-100$ or $1 \%$ digitonin before induction of disulfide linkage and analysis by non-reducing SDS-PAGE. (d) Bax $\mathrm{BH} 3$ :groove interaction is inhibited by Bcl-2. Membrane fractions from MEFs expressing Bax S55C/R94C were incubated with tBid and with increasing concentrations of recombinant $\mathrm{Bcl}-2 \Delta \mathrm{CT}$ before disulfide linkage and analysis by non-reducing SDS-PAGE. Samples were also separated to supernatant and pellet fractions for analysis of cytochrome $c$ release (lower panels). Data are representative of two independent experiments

interaction, and so provide further evidence that the two proteins adopt similar conformations during apoptosis.

To first examine whether heterodimers form in apoptotic MEFs, we used BN-PAGE, reasoning that the different a

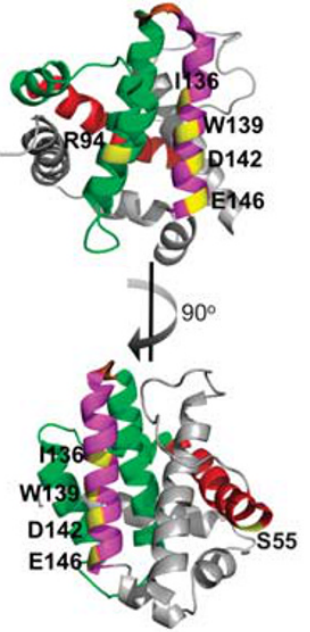

b ${ }^{100} 7^{\text {Etoposide }}$

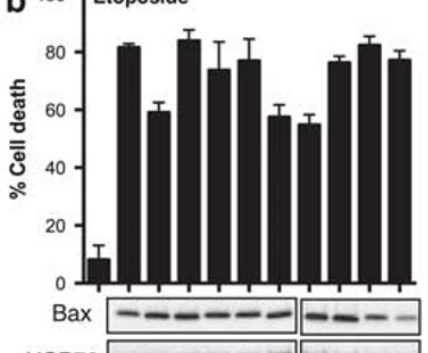

HSP70 -- - - - - -

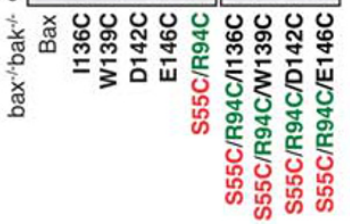

C
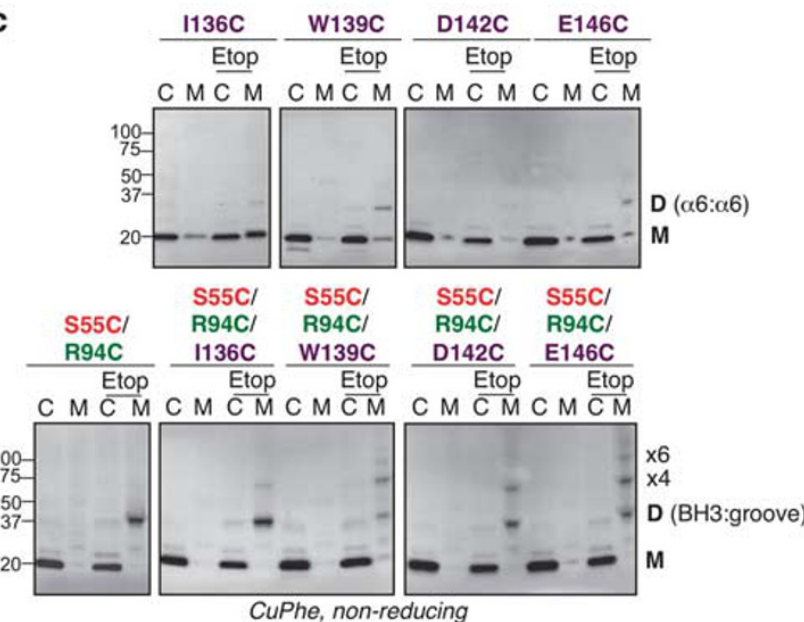

Figure 5 An $\alpha 6: \alpha 6$ interface can link symmetric BH3:groove dimers in Bax oligomers. (a) Cysteine substitutions in Bax $\alpha 6$. A cartoon representation of Bax showing residues in $\alpha 6$ (magenta), BH3 domain (red) and groove (green) that were mutated to cysteine (yellow). (b) Bax $\alpha 6$ cysteine variants retain apoptotic function. bax ${ }^{-1-} b a k^{-1-}$ MEFs expressing the indicated Bax variants were treated with etoposide $(10 \mu \mathrm{M})$. Cell death is expressed as mean \pm S.D. of three independent experiments. (c) BH3:groove dimers can link via an $\alpha 6: \alpha 6$ interface to generate higher order complexes. $b a x^{-/-} b a k^{-1-}$ MEFs expressing the indicated singledouble-, or triple-cysteine Bax variants were left untreated or treated with etoposide $(10 \mu \mathrm{M})$. Cytosol $(\mathrm{C})$ and membrane $(\mathrm{M})$ fractions were treated with CuPhe and analyzed as in Figure 1b. Samples were also run under reducing conditions (Supplementary Figure 5a). Data are representative of three independent experiments

molecular weights of Bax and Bak would distinguish heterodimers from homodimers. Mouse Bax present in mitochondrial extracts from etoposide-treated $b a k^{-1-}$ MEFs migrated as a series of discrete complexes (Figure 6a), similar to ectopically expressed human Bax (Figure 3c). If Bak was also present (wt MEFs), Bax complexes were largely unaffected except for a minor band just above the Bax:Bax dimer (Figure 6a). On the basis of size this minor band is a Bax:Bak heterodimer, and based on its low intensity it is a minor species in these apoptotic cells.

To confirm that Bak rather than some other protein was associating with Bax in this putative heterodimer band, gelshift assays using antibodies against Bak were performed. 
a

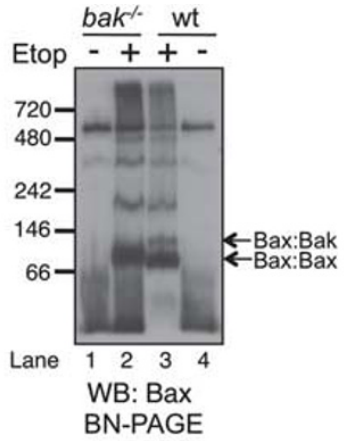

b

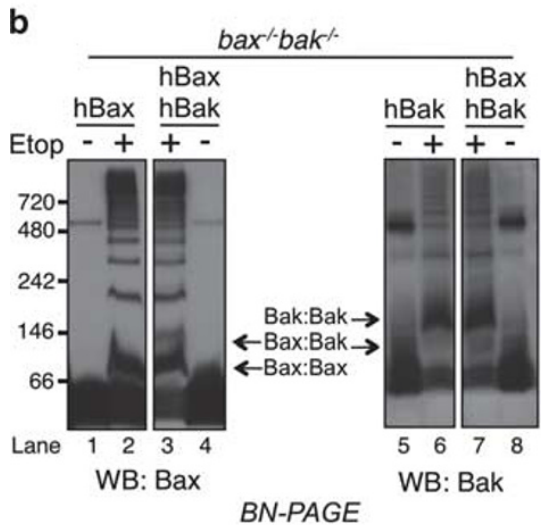

d c

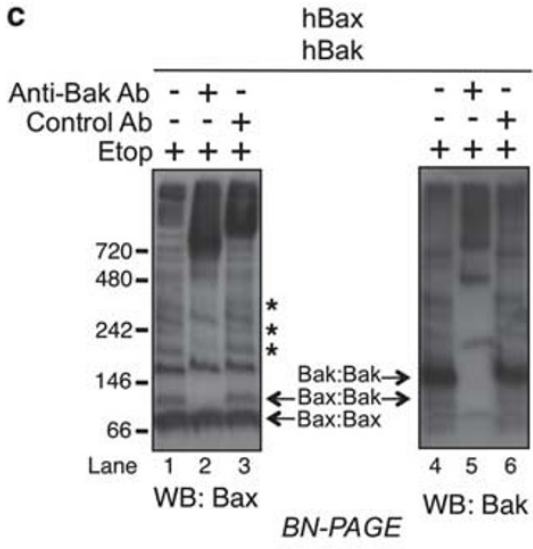

FLAG: Bak $^{\text {M71C }}$ Bax $^{\text {S55C }}{ }^{5 a x^{555 C}}$ Bak $^{\text {M71C }}$ Bax $^{\text {R94C }}$ Bak $^{\text {M71C }}$

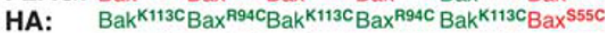

tBid $\overline{-+} \overline{-+} \overline{-+} \overline{-+} \overline{-+}$

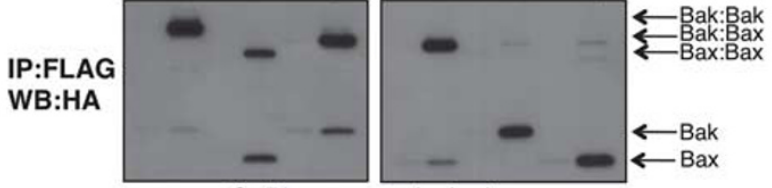

CuPhe, non-reducing)
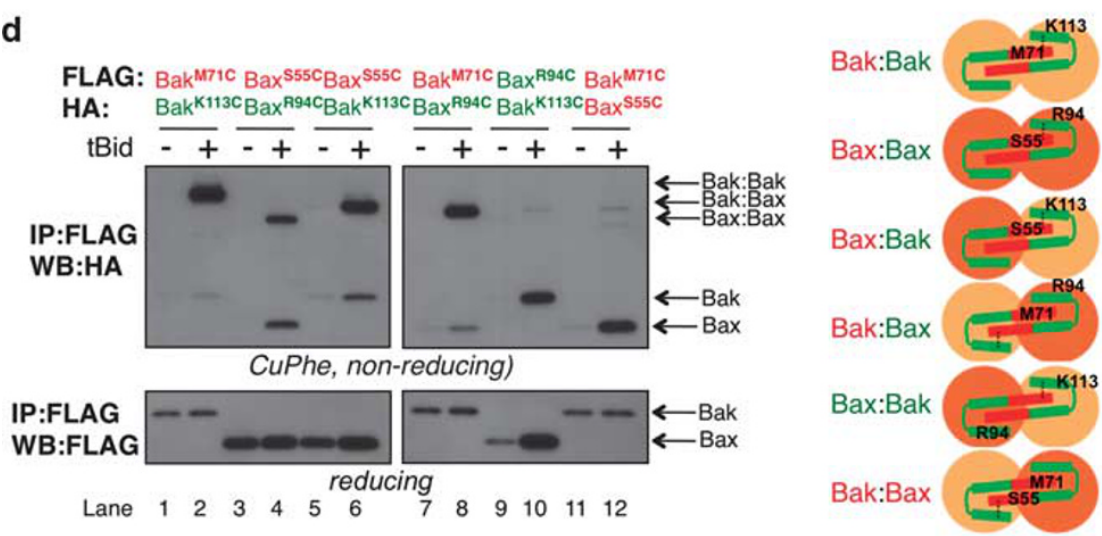

Figure 6 Bax and Bak can heterodimerize following apoptotic signaling and do so via a BH3:groove interaction. (a) Mouse Bax predominantly self-associates during apoptosis. Wild-type (wt) and bak ${ }^{-1-}$ MEFs were treated or not with etoposide for $24 \mathrm{~h}$. Membrane fractions were then run on BN-PAGE and immunoblotted for Bax. Note the appearance in wt MEFs of a band consistent in molecular weight with a Bax:Bak heterodimer. Data are representative of three independent experiments. (b) Human Bax and human Bak predominantly self-associate during apoptosis. bax ${ }^{-1}$ bak ${ }^{-1-}$ MEFs expressing hBax and/or hBak were treated with etoposide for $24 \mathrm{~h}$ and the membrane fractions run on BN-PAGE and immunoblotted for Bax or Bak. Data are representative of two independent experiments. (c) Gel-shift of Bak identifies heterodimers. bax ${ }^{-1}$ $b_{a k}{ }^{-1-}$ MEFs expressing hBax and hBak were treated with etoposide for $24 \mathrm{~h}$ as in (b). Membrane fractions were then incubated with anti-Bak antibody (7D10) or a control antibody against Bid (8C3) before BN-PAGE and immunoblotting for Bax or Bak. Data are representative of two independent experiments. *Possible higher order oligomers containing both Bax and Bak. (d) Bax and Bak associate via a BH3:groove interaction. Mitochondrial fractions were obtained from cells co-expressing the indicated variants of BaxS184L or Bak. Each variant contains a single cysteine in the BH3 domain (red) or groove (green), and each has a FLAG or HA tag. Mitochondrial fractions were left untreated or treated with tBid, and CuPhe added to induce disulfide bonds. Samples were then immunoprecipitated in 1\% digitonin for FLAG and run on non-reducing (upper panel) or reducing (lower panel) SDS-PAGE before western blotting for HA (upper panel) or for FLAG (lower panel). Also shown is a schematic of the disulfide bonding induced in each dimer of Bak (yellow) and Bax (orange). Data are representative of three independent experiments

Unfortunately, several antibodies failed to gel-shift endogenous mouse Bak (data not shown). As the most anti-Bak antibodies have been generated against the human protein, we stably expressed human Bak (hBak, FLAG-tagged) and human Bax (hBax) in bax ${ }^{-/-} b^{-1-}$ MEFs. Again, etoposide induced a putative heterodimer band when both proteins were expressed, but not when hBax was expressed alone (Figure 6b), showing that human Bax and Bak stably expressed in MEFs oligomerize in a similar fashion to the endogenous mouse proteins. Gel-shift assays on these MEFs expressing human Bax- and Bak then confirmed that Bak was present in the putative heterodimer complex (Figure 6c). 
For example, the minor Bax band was gel-shifted by antibody that recognizes all forms of Bak but not by a control antibody. A duplicate gel blotted for Bak confirmed that the anti-Bak antibody gel-shifted Bak (Figure 6c). Higher order Bax complexes migrating above the predominant Bax:Bax oligomers ( ${ }^{*}$, Figure $6 \mathrm{c}$ ) were also gel-shifted by the anti-Bak antibodies. Thus, during apoptosis a small portion of Bax and Bak heterodimerize and multimerize to high order complexes.

Cysteine linkage and co-precipitation was then used to test whether heterodimers involve a $\mathrm{BH} 3$ :groove interface. Bax and Bak cysteine variants were co-expressed with either a FLAG or HA tag, as in Figure 2. Each protein contained cysteine at equivalent positions in the BH3 domain (BaxS55C and BakM71C) or the groove (BaxR94C and BakK113C) (Figures 1a and 6d), with Bax cysteine mutants generated in the mitochondrial S184L variant to allow efficient activation of both Bak and Bax with recombinant tBid. As expected, co-expressed proteins co-precipitated only after tBid, and homodimers of Bak and of Bax efficiently disulfide-linked via a BH3:groove interaction (Figure 6d, lanes 2 and 4). Notably, heterodimers of Bak and Bax identified by their intermediate molecular weight, were also linked via a $\mathrm{BH} 3$ :groove interaction (Figure 6d, lanes 6 and 8). Indeed, BH3:groove linkage was observed in both the Bax:Bak and Bak:Bax directions, supporting a reciprocal $\mathrm{BH} 3$ :groove interaction in the heterodimers. As negative controls, disulfide-linkage within heterodimers did not occur in a $\mathrm{BH} 3: \mathrm{BH} 3$ or groove:groove manner, despite co-precipitation (Figure 6d, lanes 10 and 12).

Thus, Bax and Bak can heterodimerize during apoptosis and do so via a reciprocal BH3:groove interaction. However, as heterodimers were consistently a minor species on BN-PAGE, Bax and Bak predominantly homodimerize during apoptosis.

\section{Discussion}

These studies are the first to show that when Bax oligomerizes in apoptotic cells the $\mathrm{BH} 3$ domain binds to the hydrophobic groove, and that reciprocity of this $\mathrm{BH} 3$ :groove interaction generates symmetric dimers. The findings are consistent with recent liposome experiments in which a Bax dimerization interface involved two $\mathrm{BH} 3$ domains. ${ }^{17}$ In addition, in Bax oligomers induced by the detergent Triton $\mathrm{X}-100, \mathrm{BH} 3$ residues could form a cross-link with another Bax molecule, although the receptor surface was not identified. ${ }^{18}$

Bax is thus similar to Bak which also forms symmetric $\mathrm{BH} 3$ :groove homodimers in mitochondria of apoptotic cells, ${ }^{13,14}$ as well as in liposomes. ${ }^{27}$ The $\mathrm{BH} 3$ domain and the groove are located in the $\alpha 2-\alpha 5$ regions of both proteins, with those regions implicated by mutagenesis studies to be important for homo-oligomerization. ${ }^{13,15}$ Even at the molecular level the Bax and Bak BH3:groove interactions are similar, as near complete cysteine linkage could be induced between cysteines substituted at equivalent positions, for example, at T56 and R94C in Bax (Figure 3d) and at M71 and K113 in Bak. ${ }^{14}$

That a BH3:groove interaction in Bax is important for pore formation as well as for oligomerization is supported by several lines of evidence. First, because essentially all mitochondrial Bax adopted a BH3:groove conformation following apoptotic signaling, a $\mathrm{BH} 3$ :groove interface has to be present in those Bax oligomers responsible for MOM permeabilization rather than in a minor Bax population that might not be responsible for pore formation. Second, BH3:groove dimerization correlated with cytochrome $c$ release. Furthermore, when apoptosis was blocked by LOF mutations in the Bax $\mathrm{BH} 3$ domain or by addition of $\mathrm{Bcl}-2$, BH3:groove dimerization was also blocked.

The symmetric nature of the Bax $\mathrm{BH} 3$ :groove dimer implies that a second interface is necessary for Bax to oligomerize into the higher order complexes responsible for MOM permeabilization. In Bak, an $\alpha 6: \alpha 6$ interface could link symmetric dimers to higher order complexes. ${ }^{14}$ We now show that this also holds for Bax, although linkage at $\alpha 6$ did not trap the very high order complexes of Bax detected by BN-PAGE (Figure 3c). As $\alpha 6$ mutations performed to date did not block Bax (or Bak) function, further analysis is required to determine whether the $\alpha 6: \alpha 6$ interface is necessary for Bax (or Bak) function, or whether additional interfaces are involved. ${ }^{28}$

While heterodimerization between Bax and Bak is clearly not required for apoptosis, ${ }^{2,1}$ heterodimers are generated during apoptosis as Bax and Bak can co-precipitate, and heterodimers are evident on BN-PAGE (Figure 6). ${ }^{29,30}$ Cysteine linkage also showed that heterodimers form via a $\mathrm{BH}$ 3:groove interaction, and that the interaction was efficient in both the Bak:Bax and Bax:Bak directions, further indicating the similarity of BH3:groove interactions in Bax and Bak. However, homodimers of Bax and Bak predominate, at least as indicated by BN-PAGE, presumably due to higher binding affinities between like molecules. Heterodimers also multimerized to some degree (Figure 6c), although given the minor population of hetero-oligomers it is not possible to conclude whether they contribute to pore formation, or whether they might actually inhibit the process.

As the Bax groove acts as a receptor for the $\mathrm{BH} 3$ domain of Bax or Bak, it may also act as a receptor for $\mathrm{BH}$-only proteins such as tBid, Bim and Puma to cause 'direct' activation of $\mathrm{Bax}^{23}$ as suggested, ${ }^{28,31}$ and very recently reported for activation of Bak. ${ }^{32} \mathrm{tBid}, \mathrm{Bim}$ and Puma were reported to bind to the Bax groove, but only after first binding to an $\alpha 1 / 6$ rear pocket. ${ }^{23}$ As the Bax groove is normally occupied by the Bax transmembrane domain, that domain would need to be displaced by the activator $\mathrm{BH} 3-$ only proteins. ${ }^{4,33}$ The activator $\mathrm{BH} 3-$ only protein may itself then be displaced either by the newly exposed $\mathrm{Bax} \mathrm{BH} 3$ domain or by conformation change in the groove. This complex series of binding and conformation change is one possible explanation for the apparent transient interaction between activator $\mathrm{BH}$-only proteins and $\mathrm{Bax}$ or Bak. ${ }^{9,34}$

Is the symmetric dimer model of Bax oligomerization proposed by these studies consistent with previous studies regarding Bax oligomerization? For example, the Bax $\alpha 5 / 6$ hairpin is reported to integrate into the MOM during apoptosis, ${ }^{12}$ and as $\alpha 5$ forms part of the hydrophobic groove, ${ }^{4}$ its eversion and insertion into the MOM may disrupt the groove and preclude Bax BH3:groove dimer formation. However, our data show that the $\mathrm{BH} 3$ :groove association persists in the fully activated and presumably integrated form of Bax. Thus, loss of $\alpha 5$ from the groove may be offset by the large interacting surface area formed upon $\mathrm{BH} 3$ :groove 
binding being reciprocal. ${ }^{28}$ In terms of the sequence of Bax conformation changes, the $\mathrm{BH} 3$ :groove and $\alpha 6: \alpha 6$ interfaces may form after $\alpha 5 / 6$ inserts into the MOM, as $\alpha 5 / 6$ insertion occurs before oligomerization. ${ }^{12}$

A recent study reported that the $\mathrm{Bax} \mathrm{BH} 3$ domain binds to the $\alpha 1 / 6$ binding pocket in Triton X-100-induced Bax oligomers, ${ }^{18}$ suggesting that Bax autoactivates to form an asymmetric 'daisy chain' of monomers. ${ }^{35}$ However, our data that the $\mathrm{Bax} \mathrm{BH} 3$ domain is bound to the canonical hydrophobic groove in fully activated Bax suggest that the $B H 3: \alpha 1 / 6$ interaction may be a transient activating event, analogous to Bax activation at this site by activator $\mathrm{BH}$-only proteins. ${ }^{23,36,37}$

By defining how Bax and Bak self-associate, the current findings may help identify novel agents that block these interactions. For example, blockade of the BH3:groove interactions in Bax and in Bak may benefit conditions such as ischemic heart disease that are characterized by unwanted cell death.

\section{Materials and Methods}

Generation of cell lines and death induction. SV40-immortalized $\mathrm{bax}^{-/} \mathrm{bak}^{-/}$MEFs were retrovirally infected with human Bax or human Bak variants in internal ribosome entry site (IRES)-GFP or IRES-hygromycin expression constructs, and polyclonal populations of GFP-positive or hygromycin-resistant cells selected as previously described. ${ }^{13}$ Apoptosis was induced by incubation with etoposide $(10 \mu \mathrm{M})$ or staurosporine $(1 \mu \mathrm{M})$ for $24 \mathrm{~h}$, and the resulting cell death assessed by propidium iodide uptake. The samples were analyzed using a FACScan (Becton Dickinson, Franklin Lakes, NJ, USA). ${ }^{13}$

Mitochondrial permeabilization by tBid. For mitochondrial permeabilization assays, membrane fractions prepared by digitonin-permeabilization of untreated cells were incubated for $30 \mathrm{~min}$ at $30^{\circ} \mathrm{C}$ with $20-100 \mathrm{nM}$ recombinant Bid cleaved with caspase- 8 to generate tBid. ${ }^{13}$. Recombinant $\mathrm{Bcl}-2 \Delta \mathrm{CT}$ lacked 22 residues at the $\mathrm{C}$-terminus, and was generated as previously described. ${ }^{38}$ Supernatant $(\mathrm{S})$ and membrane pellet $(\mathrm{P})$ fractions were separated by centrifugation at $13000 \mathrm{~g}$ for $5 \mathrm{~min}$, and immunoblotted for cytochrome $c$ (clone no. 7H8.2C12, BD Pharmingen, San Jose, CA, USA).

Detecting Bax oligomerization by disulfide-linkage. Bax oligomerization in cells and in isolated mitochondria was monitored as previously described for Bak. ${ }^{13}$ Briefly, after cells were treated with etoposide in the presence of the broad range caspase inhibitor Q-VD.oph $(50 \mu \mathrm{M}$, Enzyme Systems, San Diego, CA, USA), the cell membrane was permeabilized in permeabilization buffer (20 mM HEPES/KOH pH 7.5, $100 \mathrm{mM}$ sucrose, $2.5 \mathrm{mM} \mathrm{MgCl} 2,50 \mathrm{mM} \mathrm{KCl}$, $0.025 \%$ digitonin and Complete protease inhibitors (Roche, Basel, Switzerland)). Supernatant $(S)$ and membrane pellet $(P)$ fractions were separated by centrifugation at $13000 \mathrm{~g}$ for $5 \mathrm{~min}$. To detect cysteine-linked oligomers, fractions were incubated with the oxidant copper(II)(1,10-phenanthroline $)_{3}$ (CuPhe) on ice for 15-30 min before quenching the oxidation reaction with $10 \mathrm{mM}$ EDTA and $10 \mathrm{mM}$ $\mathrm{N}$-ethylmaleimide. Where indicated, samples were incubated in $1 \%$ detergent in permeabilization buffer for $30 \mathrm{~min}$ on ice before oxidant treatment. Samples were subjected to non-reducing or reducing SDS-PAGE and immunoblotted for Bax (N-20, Santa Cruz Biotechnology, Santa Cruz, CA, USA) or Bak (aa23-38, B5929, Sigma-Aldrich, St. Louis, MO, USA).

Immunoprecipitation. Cells or cell fractions were solubilized on ice for $30 \mathrm{~min}$ in lysis buffer $(20 \mathrm{mM}$ Tris- $\mathrm{HCl}$ pH 7.4, $135 \mathrm{mM} \mathrm{NaCl}, 1.5 \mathrm{mM} \mathrm{MgCl}, 1 \mathrm{mM} \mathrm{EGTA}$, $10 \%$ glycerol) containing either $1 \%$ Triton X-100 or $1 \%$ digitonin, and anti-FLAG immunoprecipitation performed as described. ${ }^{13}$ Immunoprecipitates and cell lysates were run on SDS-PAGE under non-reducing or reducing conditions, and immunoblotted for HA (16B12, Covance, Princeton, NJ, USA) or FLAG (M2, Sigma-Aldrich). To assess Bax activation by immunoprecipitation cells were solubilized on ice for $30 \mathrm{~min}$ in lysis buffer $(20 \mathrm{mM}$ Tris- $\mathrm{HCl}$ pH 7.4, $135 \mathrm{mM} \mathrm{NaCl}$,
$1.5 \mathrm{mM} \mathrm{MgCl}, 1 \mathrm{mM}$ EGTA, $10 \%$ glycerol) containing 1\% CHAPS, and conformationally active Bax immunoprecipitated with $6 \mathrm{~A} 7$ antibody. ${ }^{5}$

BN-PAGE and antibody gel-shift assays. BN-PAGE was performed essentially as described. ${ }^{25}$ Cytosol and membrane fractions from either untreated or etoposide-treated cells were generated as above, but in the presence of $2 \mathrm{mM} \mathrm{DTT}$. Membrane fractions were then solubilized in $20 \mathrm{mM}$ Bis-tris, $\mathrm{pH} 7.4,50 \mathrm{mM} \mathrm{NaCl}$, $10 \%$ glycerol, $2 \mathrm{mM}$ DTT and $1 \%$ digitonin, before centrifugation at $13000 \mathrm{~g}$ to remove debris. 10X BN-PAGE loading dye (5\% Coomassie Blue G-250 (Bio-Rad, Hercules, CA, USA) in $500 \mathrm{mM}$ 6-aminohexanoic acid, $100 \mathrm{mM}$ Bis-tris, pH 7.0) was then added. Samples were electrophoresed on $4-13 \%$ native gels in anode buffer ( $50 \mathrm{mM}$ Bis-tris, pH 7.0) and blue cathode buffer $(50 \mathrm{mM}$ Tricine, $15 \mathrm{mM}$ Bis-tris unbuffered containing $0.02 \%$ Coomassie Blue G-250), with blue cathode buffer replaced with clear buffer (without Coomassie Blue) when the dye front was one third through the resolving gel. Gels were transferred to PVDF and immunoblotted for Bax (21C10, from DCS Huang) or for Bak (aa 23-38, B5929, Sigma-Aldrich). For gel-shift analysis, before addition of BN-PAGE loading buffer, solubilized membrane fractions from etoposide-treated cells were incubated for $30 \mathrm{~min}$ on ice with $2 \mu \mathrm{g}$ of rat monoclonal antibody against Bak $(7 D 10)^{13}$ or Bid $(8 C 3)^{39}$ as a control.

\section{Conflict of Interest}

The authors declare no conflict of interest.

Acknowledgements. We thank Peter Colman for comments on the manuscript, Michael Ryan and Michael Lazarou for advice on BN-PAGE, Stephanie Fennell and Laura Raiti for technical assistance, and David Huang and W Douglas Fairlie for reagents. The work was supported by grants from the National Health and Medical Research Council of Australia (no. 575559 and no. 637335), and the Association for International Cancer Research (no. 10-230), and operational infrastructure grants through the Australian Government IRISS and the Victorial State Government OIS.

1. Lindsten T, Ross AJ, King A, Zong W, Rathmell JC, Shiels HA et al. The combined functions of proapoptotic Bcl-2 family members Bak and Bax are essential for normal development of multiple tissues. Mol Cell 2000; 6: 1389-1399.

2. Wei MC, Zong WX, Cheng EH, Lindsten T, Panoutsakopoulou V, Ross AJ et al. Proapoptotic BAX and BAK: a requisite gateway to mitochondrial dysfunction and death. Science 2001; 292: 727-730.

3. Moldoveanu T, Liu Q, Tocilj A, Watson M, Shore G, Gehring K. The X-ray structure of a BAK homodimer reveals an inhibitory zinc binding site. Mol Cell 2006; 24: 677-688.

4. Suzuki M, Youle RJ, Tjandra N. Structure of Bax: coregulation of dimer formation and intracellular localization. Cell 2000; 103: 645-654.

5. Hsu Y-T, Youle RJ. Bax in murine thymus is a soluble monomeric protein that displays differential detergent-induced conformations. J Biol Chem 1998; 273: 10777-10783.

6. Nechushtan A, Smith CL, Hsu YT, Youle RJ. Conformation of the Bax C-terminus regulates subcellular location and cell death. EMBO J 1999; 18: 2330-2341.

7. Dai H, Meng XW, Lee SH, Schneider PA, Kaufmann SH. Context-dependent Bcl-2/Bak interactions regulate lymphoid cell apoptosis. J Biol Chem 2009; 284: 18311-18322.

8. Willis SN, Chen L, Dewson G, Wei A, Naik E, Fletcher Jl et al. Proapoptotic Bak is sequestered by $\mathrm{Mcl}-1$ and $\mathrm{Bcl}-\mathrm{xL}$, but not $\mathrm{Bcl}-2$, until displaced by $\mathrm{BH}$-only proteins. Genes Dev 2005; 19: 1294-1305.

9. Wei MC, Lindsten T, Mootha VK, Weiler S, Gross A, Ashiya M et al. tBID, a membranetargeted death ligand, oligomerizes BAK to release cytochrome c. Genes Dev 2000; 14: 2060-2071

10. Griffiths GJ, Dubrez L, Morgan CP, Jones NA, Whitehouse J, Corfe BM et al. Cell damageinduced conformational changes of the pro-apoptotic protein Bak in vivo precede the onset of apoptosis. J Cell Biol 1999; 144: 903-914.

11. Antonsson B, Montessuit S, Sanchez B, Martinou JC. Bax is present as a high molecular weight oligomer/complex in the mitochondrial membrane of apoptotic cells. J Biol Chem 2001; 276: 11615-11623.

12. Annis MG, Soucie EL, Dlugosz PJ, Cruz-Aguado JA, Penn LZ, Leber B et al. Bax forms multispanning monomers that oligomerize to permeabilize membranes during apoptosis. Embo J 2005; 24: 2096-2103.

13. Dewson G, Kratina T, Sim HW, Puthalakath H, Adams JM, Colman PM et al. To trigger apoptosis, Bak exposes its $\mathrm{BH} 3$ domain and homodimerizes via $\mathrm{BH} 3$ :groove interactions. Mol Cell 2008; 30: 369-380.

14. Dewson G, Kratina T, Czabotar P, Day CL, Adams JM, Kluck RM. Bak activation for apoptosis involves oligomerization of dimers via their alpha6 helices. Mol Cell 2009; 36: 696-703. 
15. George NM, Evans JJ, Luo X. A three-helix homo-oligomerization domain containing BH3 and $\mathrm{BH} 1$ is responsible for the apoptotic activity of Bax. Genes Dev 2007; 21: 1937-1948.

16. Simonen M, Keller $\mathrm{H}$, Heim J. The $\mathrm{BH} 3$ domain of $\mathrm{Bax}$ is sufficient for interaction of $\mathrm{Bax}$ with itself and with other family members and it is required for induction of apoptosis. Eur $J$ Biochem 1997; 249: 85-91.

17. Bleicken S, Classen M, Padmavathi PV, Ishikawa T, Zeth K, Steinhoff HJ et al. Molecular details of Bax activation, oligomerization, and membrane insertion. J Biol Chem 2010; 285 : 6636-6647.

18. Zhang Z, Zhu W, Lapolla SM, Miao Y, Shao Y, Falcone $M$ et al. Bax forms an oligomer via separate, yet interdependent, surfaces. J Biol Chem 2010; 285: 17614-17627.

19. Cheng EH, Sheiko TV, Fisher JK, Craigen WJ, Korsmeyer SJ. VDAC2 inhibits BAK activation and mitochondrial apoptosis. Science 2003; 301: 513-517.

20. Nie C, Tian C, Zhao L, Petit PX, Mehrpour M, Chen Q. Cysteine 62 of Bax is critical for its conformational activation and its proapoptotic activity in response to $\mathrm{H} 2 \mathrm{O} 2$-induced apoptosis. J Biol Chem 2008; 283: 15359-15369.

21. D'Alessio M, De Nicola M, Coppola S, Gualandi G, Pugliese L, Cerella C et al. Oxidative Bax dimerization promotes its translocation to mitochondria independently of apoptosis. FASEB J 2005; 19: 1504-1506

22. Valentijn AJ, Upton JP, Gilmore AP. Analysis of endogenous Bax complexes during apoptosis using blue native PAGE: implications for Bax activation and oligomerization. Biochem J 2008; 412: 347-357.

23. Kim H, Tu HC, Ren D, Takeuchi O, Jeffers JR, Zambetti GP et al. Stepwise activation of BAX and BAK by tBID, BIM, and PUMA initiates mitochondrial apoptosis. Mol Cell 2009; 36: 487-499.

24. Fletcher Jl, Meusburger S, Hawkins CJ, Riglar DT, Lee EF, Fairlie WD et al. Apoptosis is triggered when prosurvival Bcl-2 proteins cannot restrain Bax. Proc Natl Acad Sci USA 2008; 105: 18081-18087

25. Lazarou M, Stojanovski D, Frazier AE, Kotevski A, Dewson G, Craigen WJ et al. Inhibition of Bak activation by VDAC2 is dependent on the Bak transmembrane anchor. J Biol Chem 2010; 285: 36876-36883.

26. Ross K, Rudel T, Kozjak-Pavlovic V. TOM-independent complex formation of Bax and Bak in mammalian mitochondria during TNFalpha-induced apoptosis. Cell Death Differ 2009; 16: 697-707.

27. Oh KJ, Singh P, Lee K, Foss K, Lee S, Park M et al. Conformational changes in BAK, pore-forming proapoptotic Bcl-2 family member, upon membrane insertion and direct evidence for the existence of $\mathrm{BH} 3-\mathrm{BH} 3$ contact interface in BAK homo-oligomers. J Biol Chem 2010; 285: 28924-28937.

28. Westphal D, Dewson G, Czabotar PE, Kluck RM. Molecular biology of Bax and Bak activation and action. Biochim Biophys Acta 2011; 1813: 521-531.

29. Mikhailov V, Mikhailova M, Degenhardt K, Venkatachalam MA, White E, Saikumar $P$. Association of Bax and Bak homo-oligomers in mitochondria. Bax requirement for Bak reorganization and cytochrome $c$ release. J Biol Chem 2003; 278: 5367-5376.

30. Sundararajan R, Cuconati A, Nelson D, White E. Tumor necrosis factor-a induces Bax-Bak interaction and apoptosis, which is inhibited by adenovirus E1B 19K. J Biol Chem 2001; 276: $45120-45127$.

31. Dewson G, Kluck RM. Mechanisms by which Bak and Bax permeabilise mitochondria during apoptosis. J Cell Sci 2009; 122 (Pt 16): 2801-2808.

32. Dai H, Smith A, Meng XW, Schneider PA, Pang YP, Kaufmann SH. Transient binding of an activator $\mathrm{BH} 3$ domain to the Bak $\mathrm{BH} 3$-binding groove initiates Bak oligomerization. $\mathrm{J}$ Cell Biol 2011; 194: 39-48.

33. Schinzel A, Kaufmann T, Schuler M, Martinalbo J, Grubb D, Borner C. Conformationa control of Bax localization and apoptotic activity by Pro168. J Cell Biol 2004: 164: 1021-1032.

34. Lovell JF, Billen LP, Bindner S, Shamas-Din A, Fradin C, Leber B et al. Membrane binding by tBid initiates an ordered series of events culminating in membrane permeabilization by Bax. Cell 2008; 135: 1074-1084.

35. Shamas-Din A, Brahmbhatt $H$, Leber B, Andrews DW. BH3-only proteins: Orchestrators of apoptosis. Biochim Biophys Acta 2011; 1813: 508-520.

36. Tan C, Dlugosz PJ, Peng J, Zhang Z, Lapolla SM, Plafker SM et al. Auto-activation of the apoptosis Protein Bax Increases mitochondrial membrane permeability and Is inhibited by Bcl-2. J Biol Chem 2006; 281: 14764-147675.

37. Gavathiotis E, Suzuki M, Davis ML, Pitter K, Bird GH, Katz SG et al. BAX activation is initiated at a novel interaction site. Nature 2008; 455: 1076-1081.

38. Chen L, Willis SN, Wei A, Smith BJ, Fletcher Jl, Hinds MG et al. Differential targeting of prosurvival $\mathrm{Bcl}-2$ proteins by their $\mathrm{BH} 3-$ only ligands allows complementary apoptotic function Mol Cell 2005; 17: 393-403.

39. Kaufmann T, Tai L, Ekert PG, Huang DC, Norris F, Lindemann RK et al. The BH3-only protein bid is dispensable for DNA damage- and replicative stress-induced apoptosis or cell-cycle arrest. Cell 2007; 129: 423-433.

40. Liu X, Dai S, Zhu Y, Marrack P, Kappler JW. The structure of a Bcl-- $\mathrm{L}_{\mathrm{L}} /$ Bim fragment complex: Implications for Bim function. Immunity 2003; 19: 341-352.

\section{Supplementary Information accompanies the paper on Cell Death and Differentiation website (http://www.nature.com/cdd)}

